\title{
Die Behandlung Ohrenkranker in Bern in der ersten Hälfte des 19. Jahrhunderts
}

Markus Neiger und Urs Boschung

\section{Summary}

Ear patients were treated in Berne for the first time in 1841 at the Eye Policlinic. Professor Wilhelm Rau (1804-1861), ophthalmologist, was the founder of the first Ear Infirmary on the European continent. A diary dated from 1845 to 1863 contains information about the patients, their diagnoses and therapies. Chronic inflammations of the Eustachian tube and the middle ear were the most frequent diseases. Catheterisation of the Eustachian tube was an important tool in diagnosis and therapy.

\section{Zusammenfassung}

Ohrenkranke wurden in Bern erstmals 1841 poliklinisch im Rahmen der Augenpoliklinik behandelt. Initiator war Prof. Wilhelm Rau (1804-1861), der damit die erste Ohrenpoliklinik auf dem Kontinent schuf. Über die Patienten, die Diagnose und die Therapie ihrer Krankheit gibt ein Journal Auskunft, das von 1845 bis 1863 geführt wurde. Chronische, entzündliche Erkrankungen der Ohrtrompete und des Mittelohres werden am häufigsten diagnostiziert. Der Tubenkatheterismus spielt bei Diagnose und Therapie eine wichtige Rolle. Als Ohrentropfen wurden Metallsalze angewandt. Innerlich kamen Jodkali, schleimlösende Mittel bis zu Brechmittel zum Einsatz.

Prof. Dr. med. M. Neiger, Ahornweg 6, CH-3122 Kehrsatz - Prof. Dr. med. U. Boschung, Medizinhistorisches Institut, Bühlstrasse 26, Postfach 139, CH-3000 Bern 9 


\section{Einleitung}

Obwohl in Bern schon im 16. Jahrhundert, besonders im Inselspital, Ärzte ausgebildet wurden, wurde eine Medizinische Fakultät nach heutigem Begriff erst mit der Gründung der Universität 1834 errichtet.' Wilhelm Rau ${ }^{2}$ war der erste Professor für Augenheilkunde. Im Jahre 1841 begann er an der Poliklinik für Ophthalmiatrik auch Ohrenkranke zu behandeln.

Rau berichtet darüber in einem Brief vom 18.8.1858 an den Regierungsrat: ${ }^{3}$ «Seit dem Jahre 1841 habe ich diese Anstalt [gemeint ist die ophthalmiatrische Poliklinik] auch auf Ohrenkranke ausgedehnt und auf diese Weise die erste Ohrenklinik ins Leben gerufen, welche seither in Göttingen allein Nachahmung gefunden hat, dort aber seit dem Abgang von Prof. Ruete wieder eingegangen ist».

Diese Meinung Raus, die erste Poliklinik für Ohrenkranke gegründet zu haben, scheint sich beim Literaturstudium ${ }^{4}$ für den Kontinent zu bestätigen. So wurden nur in Irland 1814 im National Eye Hospital in Dublin und in England 1816 im Royal Ear Hospital in London sowie 1834 in der Ear Infirmary in Edinburgh Ohrenkranke behandelt. Auf dem Kontinent war es Arlt in Prag, auch Ophthalmologe wie Rau, der 1844 Ohrenkranke in der Medizinischen Abteilung von Prof. Fischer behandelte; dann folgt 1852 Leipzig, wo J. A. Winter eine private Poliklinik betrieb. Prof. Ruete (ebenfalls Ophthalmologe), der von Rau erwähnt wird, war zu dieser Zeit nach Leipzig berufen worden und hatte anfänglich seine ohrenärztliche Tätigkeit mit Winter weitergeführt. In den 60er Jahren wurden in Wien, Mailand, Halle a.S. und Berlin teils staatliche, teils private Ohrenpolikliniken eröffnet. Die eigentliche Etablierung von Polikliniken, aber auch schon von Kliniken mit einem meist sehr kleinen Bettenbestand, erfolgte in den 70er Jahren und später.

Über die Tätigkeit der «Ohrenpoliklinik» in Bern besteht im Archiv des Inselspitals ein Journal. ${ }^{5}$ Es beginnt am 8. November 1845 und endet am

1 Hochschulgeschichte Berns 1528-1984, Bern, Universität, 1984, 629.

2 Wilhelm Rau (1804-1861) wurde 1834 als a.o. Prof. für Kinderheilkunde, allgemeine Pathologie, Arzneimittellehre, Rezeptierkunst und Augenheilkunde berufen und eröffnete 1839 eine Poliklinik für Ophthalmiatrik. Bickel,M.H.:Die Schaffung neuer Lehrfächer an der Berner medizinischen Fakultät 1834-1984, und Ergänzungsband: Die Dozenten der bernischen Hochschule, in: Hochschulgeschichte Berns 1528-1984, Bern, Universität, 1984. - Staatsarchiv Bern, Hochschulakten BB IIIb, Nr. 557.

3 Staatsarchiv Bern, Hochschulakten BB IIIb Nr. 557.

4 Politzer, A.: Geschichte der Ohrenheilkunde Bd. II, 215-394. Stuttgart, 1907. Nachdruck Hildesheim, G. Olms, 1967.

Eulner, H.-H.: Die Entwicklung der medizinischen Spezialfächer an den Universitäten des deutschen Sprachgebietes (Studien zur Medizingeschichte des neunzehnten Jahrhunderts. Bd. IV), Stuttgart, Ferdinand Enke, 1970, 347-386.

5 «Ohrenkrankheiten, Poliklinik 1845-1863». Staatsarchiv Bern. Inselarchiv - Manuale und Register Nr. 335. 
23. Mai 1863. Über das Patientengut von 1841-1845 berichtet Rau selber in einer «Übersicht der in der ophthalmiatrisch-otiatrischen Poliklinik behandelten Krankheitsfälle». ${ }^{6}$

Ohrenkranke wurden um 1850 aber nicht nur an der Universitätspoliklinik behandelt. So äussert sich A. v. Tscharner ${ }^{7}$ zur Statistik der Ohrenkrankheiten in seiner Praxis ${ }^{8}$ und zur Behandlung der chronischen Trommelhöhlenentzündung?

Zum damaligen Zeitpunkt waren zwar die makroskopische Anatomie des Ohres sowie in grossen Zügen auch die Funktion des Mittelohres und der Eustachischen Trompete weitgehend bekannt. ${ }^{10}$ In seinem Lehrbuch ${ }^{11}$ beklagt sich aber Rau, dass die Kenntnisse der pathologischen Anatomie des Ohres und die Funktion des Innenohres noch mehrheitlich unerforscht seien. Auch die Untersuchungstechnik des Ohres war mangelhaft. Zwar war der Ohrtrichter schon recht weit verbreitet, der Gebrauch des Stirnspiegels aber noch nicht die Regel, wie aus dem Lehrbuch von Rau hervorgeht, obschon er den Stirnspiegel und den Gebrauch des künstlichen Lichts zur Ohruntersuchung ausgedehnt abhandelt.

Folgen wir Politzer ${ }^{12}$ und Mounier-Kuhn ${ }^{13}$, war es Toynbee, der von 1841-1855 die Grundlagen der Pathologischen Anatomie des Ohres schuf. F. v. Tröltsch sorgte 1855 für die Verbreitung des Hohlspiegels. H. Weber publizierte 1834 den Stimmgabeltest, der noch heute seinen Namen trägt, und Rinne 1855 den seinen. Die Histologie des Innenohres wurde von Corti 1851 beschrieben, 1862 berichtete Helmholtz über seine Hörtheorie. Die Funktion des Vestibularorgans wurde erst am Ende des 19. und zu Beginn des 20. Jahrhunderts bekannt.

6 Rau, W.: Übersicht der in der ophthalmiatrisch-otiatrischen Poliklinik in Bern von dem Jahre 1839-1845 behandelten Krankheitsfälle nebst Bemerkungen. Schweiz. Zeitschr.f. Med., Chir. u. Geburtsh., 1846, 300-325.

7 Abraham Franz Beat von Tscharner (1817-1894) studierte in Bern, Basel und Paris Medizin, erhielt 1840 das bernische Patent als Arzt und Wundarzt. Praktizierte auch als Spezialist für Ohren- und Halskrankheiten. Er war Gefängnisarzt, Kreisimpfarzt und Mitglied der Sanitätskommission und des Sanitätskollegiums. - Lindt, W.: Zur Erinnerung a.d. Jubiläum des hundertsten Jahrestages der Gründung der medizinisch-chirurgischen Gesellschaft des Kantons Bern. Bern, Stämpfli, 1909.

8 Tscharner,v. A. - Beitrag zur Statistik der Ohrenkrankheiten. Schweiz. Zeitschr.f. Med., Chir. u. Geburtsh., 1851, S. 283-313.

9 Tscharner,v.A.: Joddämpfe gegen chronische Trommelhöhlenentzündung. Schweiz. Zeitschr. f. Med., Chir.u.Geburtsh., 1851, 240-244.

10 Rau, W.: Über den Bau, die Verrichtungen und die Pflege des Ohres. Bern, Huber, 1859.

11 Rau,W.: Lehrbuch der Ohrenkrankheiten für Ärzte und Studierende. Berlin, H. Peters, 1856. In der Folge abgekürzt: Lehrbuch.

12 Politzer, A.: Geschichte der Ohrenheilkunde Band I. Stuttgart, 1907. Nachdruck Hildesheim, G. Olms, 1967.

13 Mounier-Kuhn, P.: Un siècle d'Oto-Rhino-Laryngologie. J. Franç. d'ORL, 32, 1983, 10-30. 


\section{Raus Poliklinik}

Nicht nur die theoretischen Grundlagen zur Behandlung Ohrkranker waren unzulänglich, auch die räumlichen Möglichkeiten und das Instrumentarium zur Untersuchung waren äusserst einfach. So stand Rau für seine Poliklinik in diesen Jahren einzig der «Schausaal» des Inselspitals ${ }^{14}$ zur Verfügung. Als Instrumentarium zur Untersuchung waren sicher verschiedene Ohrtrichter vorhanden sowie zur Durchblasung der Ohrtrompete eine Serie von Kathetern. Diesem Instrument und seiner Anwendung hatte Rau grosse Bedeutung zugemessen. ${ }^{15}$ Er hat darüber auch in der medizinisch chirurgischen Kantonalgesellschaft von Bern ${ }^{16}$ und bei der Schweiz. Naturforschenden Gesellschaft ${ }^{17}$ referiert.

Rau hatte keine Assistenten. Durchblättert man aber das zitierte Journal, stellt man fest, dass die Eintragungen von mehreren Personen gemacht wurden, während einer kürzeren Periode sogar auf französisch. In der Einleitung zu seiner Publikation von $1846^{18}$ schreibt er: «Als ein erfreuliches Zeichen ist zu erwähnen, dass bereits mehrere frühere Praktikanten der Anstalt bei dem Übertritte in die selbständige Praxis die Ohrenheilkunde mit Vorliebe ausüben». Und im Artikel über den Katheterismus der Ohrtrompete ${ }^{19}$ steht: «... indem ich den Katheterismus unter meiner Aufsicht fast immer von den Praktikanten vornehmen lasse ...» sowie ${ }^{20}$ «Ein Praktikant versuchte vor meiner Ankunft in der Poliklinik den Katheter bei einem seit längerer Zeit mit Katarrh des mittleren Ohres behafteten Manne zu appliziren ... ». Damit steht praktisch fest, dass die Eintragungen im Journal von diesen Praktikanten vorgenommen wurden, die Untersuchung und Behandlung sehr wahrscheinlich auch, meist wohl unter der Leitung Raus. Im ersten halben Jahr, d.h. vom November 1845 bis April 1846, weisen die Eintragungen einheitlich dieselbe, d.h. Raus Handschrift auf, die mit jener des Briefes, den er am 18. 8.1858 dem Regierungsrat geschrieben hat, übereinstimmt. Später muss Rau die Behandlung der ohrkranken Poliklinikpatienten mehrheitlich seinen Praktikanten überlassen haben, seine Handschrift findet sich nur noch bei

14 Siegrist, A.: Festschrift zur Eröffnung der neuen Universitäts-Augenklinik in Bern. Bern, A. Franke, 1910.

15 Rau, W.: Über den Catheterismus der Eustachischen Trompete. Schweiz. Zeitschr. f. Med., Chir. u. Geburtsh., 1845, 36-50.

16 Protokoll der Herbstversammlung der Med. Chir. Ges. Bern 1849, in der Rau über «aus Guttapercha selbst verfertigte Katheter für die Tuba Eustachii» berichtet. Schweiz. Zeitschr. f. Med., Chir. u. Geburtsh., 1849, $165 \mathrm{ff}$.

17 Verh. Schweiz. Naturforsch. Ges. Versammlung in Aarau, 1850, 66.

18 s. Anm. 6.

19 s. Anm. 15, S. 35.

20 s. Anm. 15, S. 45. 


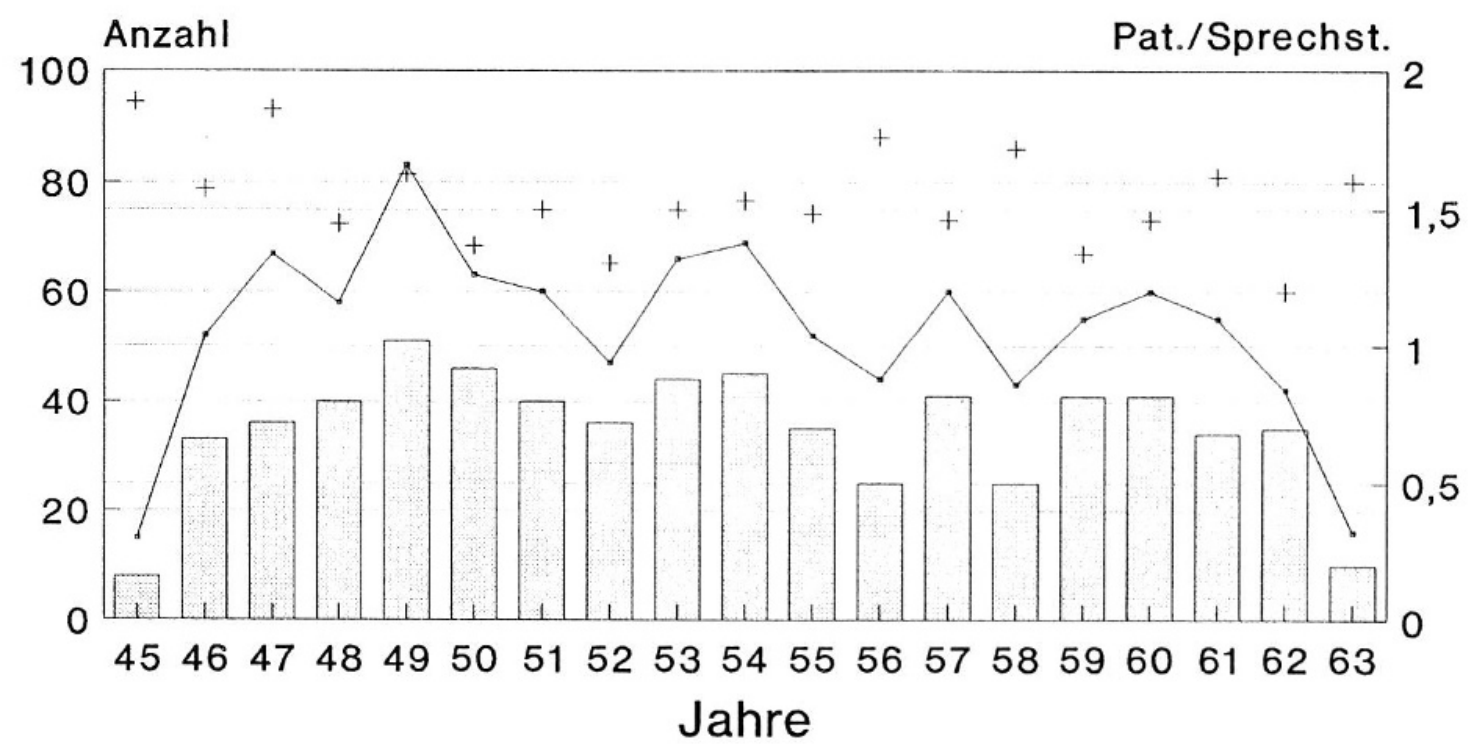

- Patienten Anzahl

$+\quad$ Neue Pat./Sprechst.

Sprechstundentermine

1845 ab Nov., 1863 bis Mai

Abb. 1. Anzahl der Sprechstunden und der Patienten der Ohrenpoliklinik Bern, von 1845-1863.

schwierigeren Fällen. Nach dem Tod Raus 1861 nimmt die Qualität der Eintragungen drastisch ab.

\section{Patienten}

Die Sprechstunden fanden in unregelmässiger Reihenfolge am Dienstag, Donnerstag und Samstag statt, sehr wahrscheinlich im Rahmen der ophthalmologischen Poliklinik, da gelegentlich Fehleintragungen über Augenkrankheiten notiert sind. Im Durchschnitt fanden in den Jahren 1846-1862 pro Jahr 38 Sprechstunden statt. Die Anzahl schwankt zwischen 25 und 51. Die Verteilung über die Jahre ist aus der Abb. 1 ersichtlich. Im ganzen wurden während dieser Jahre 1007 Patienten behandelt. Diese Zahl betrifft nur die «Neueintritte», da bei längerdauernder Behandlung die Eintragungen unter der Ersteintragung fortgeführt wurden. Wie sich die Anzahl der Patienten über die Jahre verteilt, zeigt ebenfalls Abb. 1. Aus diesen beiden Zahlen lässt sich die Anzahl Patienten pro Sprechstunde teilweise errechnen, wobei die Mehrfachkonsultationen nicht berücksichtigt sind. Durchschnittlich wurden pro Sprechstunde zwischen 1 und 2 neue Patienten untersucht (s. Abb. 1). Der jüngste behandelte Patient war 2 Monate alt, der älteste 79 


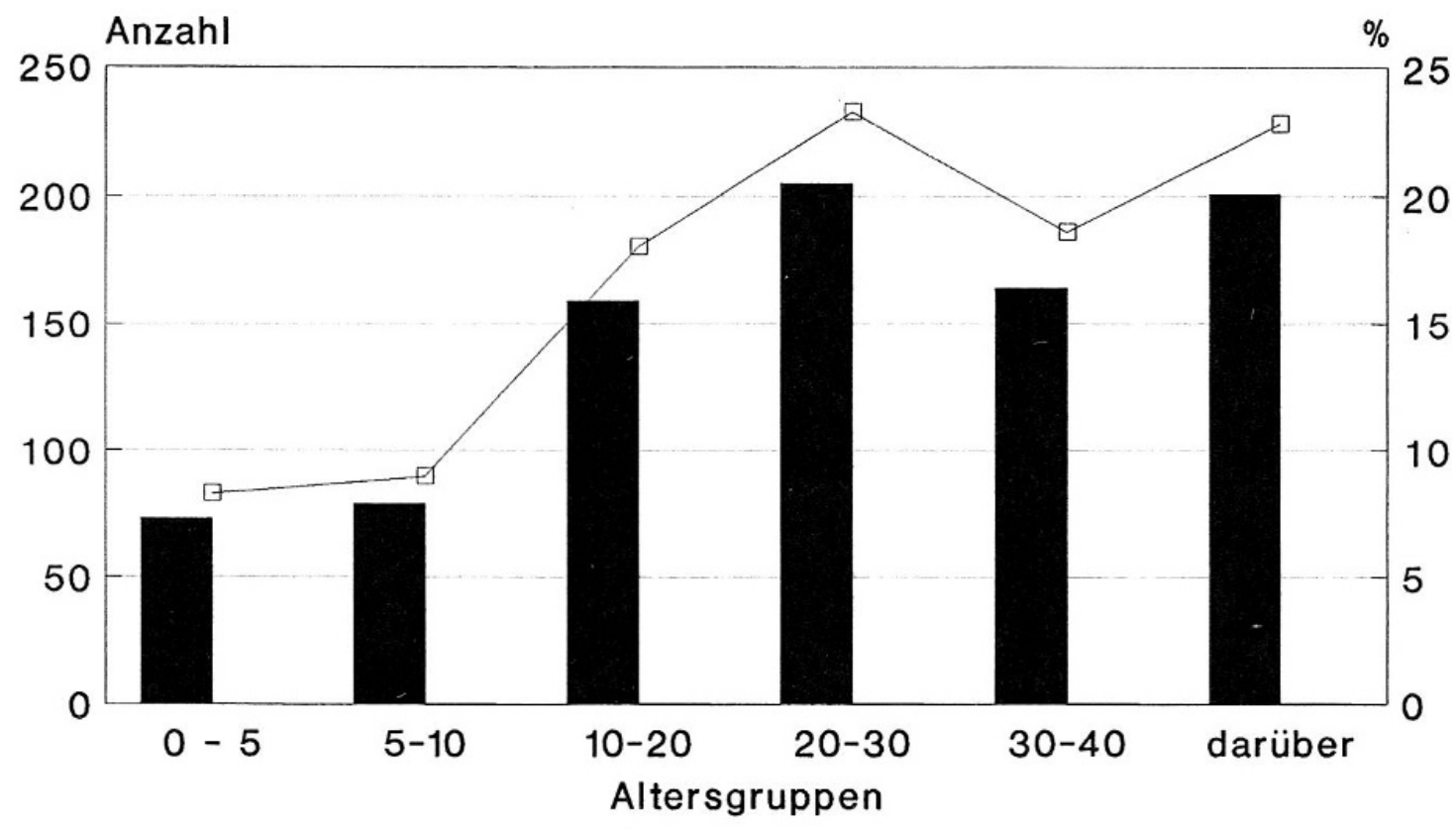

Total $\square \%$ Altersverteilung

Abb. 2. Alter der Patienten der Ohrenpoliklinik Bern von 1845-1863.

Jahre. Die Graphik (Abb. 2) zeigt einen Gipfel bei den 20-30jährigen. Auch v. Tscharner ${ }^{21}$ findet in dieser Altersklasse die meisten Patienten, schreibt er doch: « ... umfasst aber der Zeitraum vom 16ten bis zum 30sten Jahre die meisten Ohrkranken, nämlich 1/3 derselben ... ». Prozentual sind allerdings die über 40jährigen in unserer Statistik fast ebenso stark vertreten, während bei v. Tscharner die 30-80jährigen einen Drittel ausmachen.

Vergleicht man diese Zahlen mit den von Rau publizierten ${ }^{22}$, sieht man, dass in den Jahren 1841 und 1842 nur 9 Patienten wegen Ohrenleiden die Poliklinik aufgesucht haben, dass es im Jahre 1845 aber schon deren 57 waren. Im ganzen sind von 1841-1845 136 Patienten behandelt worden.

Ein genauer Vergleich mit den Zahlen v. Tscharners lässt sich nicht anstellen. Er berichtet über eine «ununterbrochene Reihenfolge von 200 von mir untersuchten Ohrenkranken», sagt aber nicht in welchem Zeitraum. Da der Artikel 1849 erschienen ist und v. Tscharner 1841 zu praktizieren begann, darf man höchstens einen Zeitraum von rund 6 Jahren annehmen. Von 1841 bis 1849 wurden in der Poliklinik rund 400 Patienten untersucht und behandelt. 


\section{Untersuchungsmethoden}

Zur Untersuchung der Patienten gehörte sicher eine Otoskopie mit dem Ohrentrichter, damals Ohrenspiegel genannt, aber kaum die Benützung des Hohlspiegels, obschon Rau diesen in seinem Lehrbuch erwähnt. Vielmehr wurde der Patient so gesetzt, dass Sonnen- oder künstliches Licht in einem günstigen Winkel auf das Ohr schien und dass durch Ziehen der Ohrmuschel nach hinten oben der Lichteinfall bis gegen das Trommelfell reichte. Zur Untersuchung gehörte routinemässig auch eine Inspektion des Rachens. So schreibt Rau: ${ }^{23}$ « ... versäume man aber niemals, in allen Fällen, so eine Krankheit der Tube und der Paukenhöhle zu ermitteln ist, eine genaue Untersuchung der inneren Theile des Halses und der Nase vorzunehmen ... ». Erwähnungen über Erkrankungen der Nase sind im Journal allerdings keine zu finden.

Das Gehör wurde mit einer Taschen- oder Repetieruhr getestet. So lesen wir: «Sie hörte den Schlag einer Repetieruhr auf dem Ohr nicht, nicht einmal die Vibrationen. Nach Catheterismus wird die Uhr auf eine Entfernung von 5-6 Zoll gehört». Auch der Test von Weber muss bekannt gewesen sein, wurde von $\mathrm{Rau}^{24}$ aber abgelehnt, weil «der Arzt die subjektiven Empfindungen des Kranken nicht controlliren kann». Trotzdem steht bei einem Patienten: «Der Ton der Stimmgabel wird auf die Stirne gesetzt nicht gehört, wohl aber auf dem proc. mastoid., ebenfalls ein wenig auf dem linken Ohr, wenn die Gabel auf den Nasenrücken gesetzt wurde». Rau hat auch mit einer «Art Repetieruhr» ein Instrument zur Gehörmessung konstruiert. ${ }^{25}$

Bei Verdacht auf Tubenbelüftungsstörungen war die Luftdusche als diagnostische Massnahme die Regel, um «eine gestörte Schalleitung von einer gestörten Funktion des Hörnerven» unterscheiden zu können, so Rau. ${ }^{26}$

\section{Diagnosen}

Von den 136 in den Jahren 1841-1845 in der Poliklinik behandelten Patienten $^{27}$ hatten 63 Probleme mit der Ohrtrompete. Überhaupt scheinen Ent-

23 Lehrbuch, 43.

24 Lehrbuch, 37.

25 s. Anm. 17, S. 66 ... «es ist dies eine Art Repetieruhr, an welcher man den Ton der Glocke nach bestimmten Graden dämpfen und den Hammer schneller oder langsamer schlagen lassen, auch das Schlagen ganz einstellen kann .... spricht ihm einen bedeutenden Vorrang vor dem gewöhnlichen Gebrauch der Stimmgabel zu, welche er als unsicher bezeichnet».

26 Lehrbuch, 61.

27 s. Anm. 6, S. 304. 
zündungen der Mittelohrräume ein Hauptgrund gewesen zu sein, den Ohrenarzt aufzusuchen ${ }^{28}$. So ist auch im Poliklinikjournal bis ins Jahr 1850 die Diagnose «chronischer Tubenkatarrh» am häufigsten anzutreffen. Allerdings lässt sich die Diagnose wegen lückenhafter Eintragungen der Befunde meist nicht verifizieren. Das Journal hält in einer Rubrik nur Name, Vorname und Alter des Patienten fest sowie meist in lateinisch die Diagnose. In einer zweiten Rubrik sind eine kurze Anamnese und die Therapie notiert. Befunde vom Gehörgang oder Trommelfell oder die Hörfähigkeit sind nur ganz selten festgehalten.

Weitere häufige Diagnosen lauten auf Myringitis, Otitis scrophulosa und Otorhoe. Später finden sich auch Cerumen, Otitis interna (heute media), Trommelfellruptur, Gehörgangspolyp, Otitis mit Kongestion des Mastoids, Dysecoia, meist congenita, für Perzeptionsschwerhörigkeit oder Taubheit. Zudem sind auch Lymphadenitiden, Angina, Hypertrophie der Mandeln und katarrhalische Entzündungen des Halses erwähnt.

Im Juli/August 1857 wird über eine «myringitis scarlatinosa» und eine «otorhoe post scarlatinam» berichtet sowie über einen Fall mit Otitis und Fazialislähmung. Die Frage, ob eine Scharlachepidemie stattgefunden hatte, drängt sich geradezu auf.

Interessant sind aber auch Diagnosen, die selten oder nur einmal erwähnt werden, wie «surditas e meningitide», «catarrhus auris media cum angina syphilitica», «commotio cerebri cum haemorrhagia ex aure».

Mit dem aus heutiger Sicht eigentlich bescheidenen Wissen und vor allem den sehr beschränkten Untersuchungsmöglichkeiten zeigen diese Diagnosen doch, dass in Bern der Beginn der modernen Otiatrik Einzug gehalten hatte.

\section{Therapie}

Die Therapiemöglichkeiten hingegen waren an der Poliklinik bescheiden. Vor allem findet man keine Indikationen zur chirurgischen Behandlung der Mastoiditis; die Parazentese wird bei der akuten Mittelohrentzündung nicht erwähnt. Dabei haben die prekären Raumverhältnisse, das Fehlen eigener Spitalbetten, sicher eine Rolle gespielt. Über die Entwicklung der Ohrpa-

28 Lehrbuch, 221, «Unter 1000 bis zum Jahr 1850 von mir behandelten Ohrkrankheiten betrug die Zahl der Schleimhautentzündungen des mittleren Ohres 352, ein Verhältnis, welches mit den Angaben v. Tscharners [loc. cit. Anm. 8], nach welcher unter 200 Kranken die Eustachische Trompete 69 mal entzündet war, wesentlich übereinstimmt. Es ist dies um so bemerkenswerther, da beide Resultate in dem gleichen Wirkungskreis gewonnen wurden.» 
thologie und der therapeutischen Möglichkeiten, die um diese Zeit herum zur modernen Otologie geführt haben, muss Rau aber orientiert gewesen sein, betrachtet man die ausgedehnte und neuzeitliche Literatur, die er in seinem Lehrbuch 1856 anführt. Sucht man in den Jahresberichten des Inselspitals $^{29}$, so findet man erst 1888 in der Operationsstatistik der nichtklinischen chirurgischen Abteilung ${ }^{30}$ den Vermerk:Trepanation des Mastoids 4 mal, von Prof. Niehans ${ }^{31}$ operiert. Ob Rau in seiner Privatpraxis Operationen am Ohr durchgeführt hat, ist nicht eruierbar.

Hingegen werden, wenn auch selten, Gehörgangspolypen entfernt, sei es durch Unterbindung des Stiels oder mit einer Schlinge. Ein Kirschkern im Gehörgang wird mit einer umgebogenen Nadel extrahiert, und einmal findet man den Vermerk, dass die rechte Mandel wegen Otitis «interna» entfernt worden sei. Wegen «surditas completa» wurde einmal eine Trommelfellperforation angelegt..$^{32}$

Vergleicht man die therapeutischen Angaben im Journal mit den Empfehlungen Raus in seinem Lehrbuch, so kann man eine weitgehende Übereinstimmung feststellen, mit einer Ausnahme. Für die Behandlung des chronischen Tubenkatarrhs oder chronischer Mittelohrentzündungen benützt er in seiner Privatpraxis Insufflationen über den Tubenkatheter mit einer «Compressionspumpe» und bläst Essigäther, Kohlensäure, Jodwasserstoffätherdämpfe ${ }^{33}$ und Harze ins Mittelohr, gemäss seinem Grundsatz: «Der Catheterismus der Eustachischen Röhre ist das einzige zuverlässige Mittel, direct auf das mittlere Ohr einzuwirken $»^{34}$; er bemerkt aber, dass dies in der Poliklinik der dürftigen Platzverhältnisse wegen nicht möglich ist. Auch v.

29 Jahresbericht der Inselspitaldirektion pro 1888, erstattet an die Inselspitalverwaltung zu Handen des Regierungsraths des Kantons Bern. Bern, G. Michel, Staatsarchiv Bern, St.A.B. PB 170.

30 Nichtklinische chirurgische Abteilung: Bedeutung dieses Ausdrucks siehe E. Hintzsche in: Rennefahrt H. und Hintzsche E.: Sechshundert Jahre Inselspital 1354-1954, verfasst im Auftrag der Inselkorporation, Bern, Hans Huber, 1954.

31 Niehans, Paul (1884-1912), «Inselarzt» 1880-1909, Leiter der nichtklinisch chirurgischen Abteilung des Inselspitals. - Die Dozenten der bernischen Hochschule. Ergänzungsband: Hochschulgeschichte Berns 1528-1984, Bern, Universität, 1984.

32 S.Anm. 5, Eintrag Nr. 43, 1853 «Locher Michel,30 J.Surditas completa. Der mit concrementen angefüllte Gehörgang wird mit Wasser ausgespritzt. Nachdem die Stimmgabel zu wiederholten Malen umsonst angewandt wurde, um die Integrität des Hörnerven zu prüfen, u. also die Prognose von vorne herein als nicht günstig qualifizirt wurde, so wurde mit einem eigens dazu verfertigten Instrument (der gewöhnliche Trepan mit schraubenförmigem Bohrer in der Canüle, um das Tympan. bei der Operation entgegen zu halten) die Perforation des Trommelfelles unterhalb des Hammerstielansatzes, vollzogen. Bald war mit einem knackenden Geräusch ein kreisrundes Stück aus dem Trommelfell geschnitten. Das Gehör blieb aber nach der Operation wie vorher stumpf .... . Der Schmerz bei der Operation war unbedeutend».

33 Jodwasserstoffäther = Äthyljodid. - Realencyclopädie der gesamten Pharmacie, Bd. 1, Wien Leipzig, 1880, 55.

34 Lehrbuch, 114. 
Tscharner ${ }^{35}$ empfiehlt die Applikation von Joddämpfen bei chronischen, von Wasserdampf und Luft bei eher akuten Zuständen.

Als Ohrentropfen kamen Metallsalze wie «zincum sulfuricum», «cuprum sulfuricum», «plumbum aceticum» sowie «solutio sachari saturni», meist kombiniert mit Opium, zur Anwendung, dies, obschon Rau vor Opium wegen der Erzeugung von Somnolenz warnte. Auch Mandelöl und «oleum jecoris» wurden bei trockenem Gehörgang verschrieben, «um die Ohrschmalzabsonderung wiederherzustellen».

Neben dem «Catheterismus» war bei Affektionen der Ohrtrompete und der Paukenhöhle regelmässig die Behandlung des «Catarrhs des Halses» üblich, so mit Salmiak, Lugolmixtur und Alaun zum Gurgeln, ferner Jodkali, Salmiak mit «tartarus stibiatus», «solutio ammonii chlorati» und «succus liquiritiae», ferner «aqua menthae piperitae» zum Einnehmen.

Bei fliessenden Ohren wurden selbstverständlich immer Tropfen verordnet.

Wurde ferner eine «Dyscrasie» festgestellt, gesellte sich zur Behandlung des lokalen Leidens Calomel mit «sulfur auratum», dann aber auch Ipecacuanha und Kermes. ${ }^{36}$

Griff die Entzündung des Mittelohres auf das Mastoid über, wurden Blutegel angesetzt oder Kataplasmen aufgelegt, um den Abszess «zum Reifen» zu bringen.

Die Behandlung eines chronischen Tubenkatarrhs konnte sich über Monate hinziehen ${ }^{37}$, rasche Heilungen waren aber auch zu verzeichnen. ${ }^{38}$

35 s. Anm 9.

36 Dyskrasie:Anomalie der Säfte, bzw. des Blutes, schlechter Ernährungszustand und schlechte Heilungstendenz. Die Säftelehre Galens war noch nicht ganz überwunden. Kermes: Stibium sulfuratum rubeum (Brechweinstein in Verbindung zu rad. Ipecacuanha - zur Entleerung des Magens oder als Resolvens bei Bronchial- und Lungenaffektionen). Real-Encyclopädie der gesamten Heilkunde, 2. Aufl., Bd. 1, Wien, Urban u. Schwarzenberg, 1885, 514.

37 s. Anm. 5, Eintrag von Donnerstag 5. März 1846 «Frutig Christian 36 J. (Catarrh chron. Tub. Eustachii). Von Kindheit an schwerhörig. Seit einigen Monaten Zunahme der Beschwerden in Folge einer angina catarrhalis. Noch jetzt Geschwulst der Mandeln. Angeblich früher Ausfluss aus dem rechten Ohr, wovon jetzt keine Spur bemerkbar. Ord. Salmiak mit tart. stib., Gurgelwasser von Alaun.

19. März - Gehör etwas besser. Rep.

31 März Rep.

11 April Rep.

28. April - Das linke Ohr zeigt sich für die Luftdusche zugänglicher als das rechte. Repet. Sulph. aur. c. Ipecae, Gargarismen Alaun

12. Mai - Besserung des Gehörs

19. Mai - Digitalis in Infus.

26. Mai - Allg. Unwohlsein, Herzklopfen. Gehör wenig geändert. Ord. Repet.

9. Juni - Kermespillen

23. Juni - Besserung des Gehörs».

38 s. Anm. 5, Eintrag von Samstag, 20. Juli 1959 «Häubi Maria 45 J. (Obturatio tubae Eustachii). Leidet seit 8 Jahren an Hemikranie, Prosopalgie, dabei an vollkommener Taubheit des lin- 
Bei der sogenannten «nervösen Schwerhörigkeit», auch Dysecoia genannt, war schon die Diagnose unsicher ${ }^{39}$, für den Nicht-Spezialisten vor allem der Unterschied zwischen Transmissions- und Perzeptionsschwerhörigkeit. So empfiehlt $\mathrm{Rau}^{40}$ «keine Schwerhörigkeit als nervöse zu behandeln, so lange materielle, entfernbare, wenn auch scheinbar zufällige Veränderungen vorhanden sind, welche für sich eine Gehörstörung bedingen können». In der Poliklinik wurden solche Schwerhörigkeiten mit «extractum belladonnae» und «aqua laurocerasi» behandelt; vermutete man eine Lues, erfolgte eine Quecksilbertherapie bis zum Auftreten einer Salivation. In der Privatpraxis wurden auch über den Tubenkatheter Dämpfe von Schwefelund Essigäther sowie Chloroform, Castoreumtinktur, Mastix und Benzoe ins Mittelohr geleitet ${ }^{41}$, je nachdem, ob es sich um eine «torpide» oder «erethische» Form gehandelt hatte, um den Hörnerven «anzuregen» oder «zu beruhigen».

Überschaut man die Therapie dieser Jahre, so ist zu bedenken, dass die pathologisch-anatomischen Kenntnisse weitgehend fehlten und die Untersuchungsmethoden rudimentär waren. Auch herrschten sehr prekäre Raumverhältnisse. Die «Inhalationsbehandlung» durch den Tubenkatheter war deshalb nicht möglich, und auch die kleinste «chirurgische» Intervention war ausgeschlossen. So wurde eine Mastoiditis noch mit Blutegeln und Kataplasmen «zum Reifen» gebracht. Aber auch das «Purgieren» war noch nicht ganz überwunden. $\mathrm{Ob}$ all dem darf man allerdings nicht vergessen, dass die fachspezifische Behandlung von Ohrenleiden erst am Anfang war und die stürmische Entwicklung der Ohrchirurgie erst nach dem Tode Raus einsetzte.

ken Ohres, die wahrscheinlich mit dieser Neuralgie in Verbindung sind. Sie hört den Schlag einer Repetieruhr auf das Ohr gehalten gar nicht, nicht einmal die Vibrationen. Um die Diagnose festzustellen, macht man die Catheterisation der Tuba Eustachii, worauf sie die Repetieruhr deutlich in einer Entfernung von 5-6 Zoll, im Verlauf von 1/4 Stunden aber auf 8 Zoll hört. Ord. Extr. belladon. gr XII, Aq. Laurocera. zi, 4xtgl. 20 Tropfen.

25.7. - Das Gehör ist geblieben, Luftduschen».

39 Rau,W.: Über die nervöse Schwerhörigkeit und deren Localbehandlung, nebst Beschreibung einer Brillenpincette zur Fixierung des in die Eustachische Röhre gebrachten Katheters. Schmidt's Jahrbücher der in- und ausländischen Medizin, 65, 1850, 250-256.

40 s. Anm. 39, S. 251.

41 s. Anm. 39, S. 252. 